Revue de l'Institut des langues et cultures

d'Europe, Amérique, Afrique, Asie et Australie

$41 \mid 2020$

Escrituras nómadas en el mundo hispánico contemporáneo

\title{
Fronteras, espacios híbridos, escrituras mutantes en Norte de Edmundo Paz Soldán y Tukzon, historias colaterales de Giovanna Rivero
}

Frontières, espaces hybrides et écritures en mutation dans Norte d'Edmundo

Paz Soldán et Tukzon, historias colaterales de Giovanna Rivero

Frontiers, Hybrid Spaces and Mutant Writings in Edmundo Paz Soldán's Norte and Giovanna Rivero's Tukzon, historias colaterales

\section{Erich Fisbach}

\section{OpenEdition}

Journals

Edición electrónica

URL: http://journals.openedition.org/ilcea/11012

DOI: 10.4000/ilcea.11012

ISSN: 2101-0609

Editor

UGA Éditions/Université Grenoble Alpes

Edición impresa

ISBN: 978-2-37747-224-6

ISSN: 1639-6073

\section{Referencia electrónica}

Erich Fisbach, «Fronteras, espacios híbridos, escrituras mutantes en Norte de Edmundo Paz Soldán y Tukzon, historias colaterales de Giovanna Rivero», ILCEA [En línea], 41 | 2020, Publicado el 03 noviembre 2020, consultado el 03 febrero 2021. URL: http://journals.openedition.org/ilcea/11012 ; DOI: https:// doi.org/10.4000/ilcea.11012

Este documento fue generado automáticamente el 3 febrero 2021.

(C) ILCEA 


\title{
Fronteras, espacios híbridos, escrituras mutantes en Norte de Edmundo Paz Soldán y Tukzon, historias colaterales de Giovanna Rivero
}

\author{
Frontières, espaces hybrides et écritures en mutation dans Norte d'Edmundo \\ Paz Soldán et Tukzon, historias colaterales de Giovanna Rivero \\ Frontiers, Hybrid Spaces and Mutant Writings in Edmundo Paz Soldán's Norte \\ and Giovanna Rivero's Tukzon, historias colaterales
}

\section{Erich Fisbach}

1 Desde la recuperación de la democracia en Bolivia en 1982 tras décadas de gobiernos autoritarios, la imagen de una literatura encerrada en sus fronteras, condicionada por la geografía del país, se diluye. En épocas anteriores, los novelistas raras veces se alejaban en sus obras de esa geografía, e incluso se podría decir que la reivindicación de un espacio propio era un elemento de afirmación identitaria. Podríamos citar aquí el ejemplo de Néstor Taboada Terán, un novelista, cuentista, periodista que tuvo que exiliarse en Argentina durante la dictadura del general Hugo Bánzer (1971-1978), después de que sus libros fueran quemados en la plaza 14 de septiembre de Cochabamba en 1972 y después de haber sido encarcelado varios meses. Estando exiliado, Taboada Terán publicó su novela más famosa, Manchay Puytu, el amor que quiso ocultar Dios, ganadora de la Faja de Honor de la Sociedad Argentina de Escritores (SADE) en 1978 (2001). Como para reivindicar su identidad, su bolivianidad, Taboada Terán trasladó la trama de la novela en la Potosí del siglo xviII, es decir a la vez en un ámbito histórico, cultural, y en un marco espacial que se identifican plena y fundamentalmente con Bolivia, cuando poco más de un siglo antes, el peruano Ricardo Palma situaba por su parte esta misma leyenda en la región de Cuzco (1964). El alejamiento forzado de 
Néstor Taboada Terán, el exilio político, derivan así en una obra que se arraiga en lo más hondo de la historia y de la geografía del país.

2 La realidad es muy distinta a partir de los últimos años del siglo pasado y no es específica de los escritores bolivianos sino que la comparte la mayoría de los escritores del continente; como lo escribe a este respecto Francisca Noguerol refiriéndose a la narrativa hispanoamericana:

[...] los años noventa han visto la aparición de una hornada de escritores cosmopolitas por biografía y vocación, comprometidos con su carrera literaria y dispuestos a desplazarse a otros países para alcanzar proyección internacional. Deseosos de romper con los estereotipos sobre el escritor latinoamericano, estos autores retratan en sus textos sociedades multiculturales, caóticas y tecnificadas en las que cada vez resulta más evidente la manipulación de la verdad (2008:27).

Vemos así que desde los años 1990, los novelistas bolivianos participan plenamente de la llamada globalización cultural, expresión con la que se designa el proceso mediante el cual los medios de comunicación conllevan una fusión cultural que tiende a borrar las particularidades y las fronteras. En una tribuna publicada en el periódico El País en abril de 2000, unos años antes de que se generalizaran las redes sociales, Mario Vargas Llosa hacía una descripción apocalíptica del proceso de globalización, vector de uniformización y de empobrecimiento cultural:

La desaparición de las fronteras nacionales y el establecimiento de un mundo interconectado por los mercados internacionales infligirá un golpe de muerte a las culturas regionales y nacionales, a las tradiciones, costumbres, mitologías y patrones de comportamiento que determinan la identidad cultural de cada comunidad o país. Incapaces de resistir la invasión de productos culturales de los países desarrollados $-\mathrm{o}$, mejor dicho, del superpoder, los Estados Unidos-, que, inevitablemente, acompañan como una estela a las grandes trasnacionales, la cultura norteamericana (algunos arrogantes la llaman la «subcultura») terminará por imponerse, uniformizando al mundo entero, y aniquilando la rica floración de diversas culturas que todavía ostenta (2000).

Demás está decir que, a veinte años de este pronóstico desalentador, la globalización, la porosidad de las fronteras, la circulación de las personas, han generado nuevas formas narrativas, nuevas prácticas culturales que aparecen como factores de renovación narrativa como lo podemos observar con la actual generación de escritores bolivianos.

5 Tanto Giovanna Rivero, nacida en Santa Cruz en 1972, como Edmundo Paz Soldán, nacido en Cochabamba en 1967, residen desde hace ya varios, por no decir muchos años fuera de su país de origen, Bolivia. Giovanna Rivero se radicó efectivamente en los Estados Unidos hace más diez años (Matto, 2016), mientras que, por su parte, Edmundo Paz Soldán vive en los Estados Unidos desde 1991, año en que se licenció en la universidad de Alabama, en Huntsville -localidad en la que se produce el desenlace de la novela Norte-, antes de obtener un doctorado en la universidad de Berkeley (1997) y luego un cargo de profesor de literatura latinoamericana en la universidad de Cornell.

En un texto titulado «Berlín, Santa Cruz» publicado en 2012 por la editorial boliviana El Cuervo, Giovanna Rivero escribe:

Uno no aprende tanto al irse sino al volver. Cosas sencillas, claro, pequeñas como la cabeza de un alfiler, pero con las que podrías atragantarte, atorarte en las aduanas de la vida. Yo me he vuelto y me he largado muchas veces y esto es un poco la crónica pendular de esos viajes sin misterio (2012: 205).

7 Por su parte, en «Tres postales», publicado en el mismo volumen, Edmundo Paz Soldán cuenta cómo, a principios de nuestro siglo, cuando ya era profesor en la Universidad 
norteamericana de Cornell, llevó a sus estudiantes a que conocieran Bolivia, en una época de protestas contra la política neoliberal del entonces presidente Gonzalo Sánchez de Lozada. Cuenta entonces cómo se encontró con sus estudiantes en medio de una violenta manifestación de indios aimaras y cómo tuvo pues la impresión de ser «un guía perdido en [su] propio país» (2012: 205).

8 Edmundo Paz Soldán y Giovanna Rivero forman pues parte de aquellos escritores que eligieron residir fuera de su país, que abordan y desarrollan desde allí temáticas muy alejadas del localismo o del regionalismo tradicionales, y que no se ciñen por consiguiente a ningún límite geográfico nacional. Todos ellos participan sin lugar a duda de ese momento que vive la narrativa latinoamericana, «un momento en que la búsqueda de identidad ha sido relegada a favor de la diversidad», como lo escribe Francisca Noguerol, afirmando que la consecuencia es que «la creación literaria se revela ajena al prurito nacionalista a partir del cual se la analizó desde la época de la Independencia...» (2008: 20), fenómeno que Francisca Noguerol define como extraterritorialidad. Ahora bien, nos parece importante subrayar aquí que la extraterritorialidad de los autores a los que nos referimos más particularmente, no es exactamente la que George Steiner aplicaba a ciertos autores como Nabokov o Wilde, que derivaba de la relación particular de estos escritores con la lengua y que permitiría analizar problemas como «el de la imaginación multilingüe, la traducción interiorizada y la posible existencia de un idioma mixto "subterráneo", "preexistente" a la localización de diversas lenguas en el cerebro articulado» (2002: 24). Los novelistas bolivianos que escriben desde fuera de Bolivia lo hacen efectivamente en español, de modo que en su caso la extraterritorialidad puede darse tanto por el marco geográfico de sus obras como por los temas desarrollados que se apartan de su tierra de origen.

La octava novela de Edmundo Paz Soldán, Los vivos y los muertos (2009), es en ese sentido un ejemplo muy simbólico. La trama transcurre en efecto en un espacio en el que se nombran varias localidades norteamericanas y al que, por una larga serie de rasgos culturales, reconocemos como norteamericano, aunque la ciudad que constituye el marco principal del relato, Madison, sea ficticia. Pero al mismo tiempo ningún personaje es boliviano, ni siquiera latinoamericano, con lo cual los únicos vínculos con Bolivia son la lengua de la novela, que no coincide con el idioma con el que comunican los personajes, así como algunas referencias o expresiones intertextuales que nos remiten a Bolivia, pero de las que no tienen ninguna conciencia los personajes. Pensemos por ejemplo en la expresión «sacarme el cuerpo» que Paz Soldán pone en boca de Amanda y que nos remite a la novela de Jaime Sáenz Felipe Delgado ${ }^{1}$ (2009: 105), o en aquella otra expresión en boca esta vez de Daniel, «La veía casi como a mi hija, la niña de mis ojos», que nos remite indudablemente a la novela de Antonio Díaz Villamil, La niña de sus ojos ${ }^{2}$ (2009: 171). Los vivos y los muertos es pues un claro ejemplo de la extraterritorialidad de cierta narrativa boliviana en la medida en que los únicos elementos que vinculan al fin de cuentas la narración con Bolivia son el nombre del autor y la lengua que emplea, aunque ésta no sea la lengua en la que piensan y comunican los personajes. Vemos así que la trayectoria personal y creativa de Giovanna Rivero y de Edmundo Paz Soldán es emblemática de esta evolución de la literatura escrita por bolivianos. Esto es lo que se destaca de varias reseñas como la que publicó Ernesto Calabuig en la revista española El Cultural del 29 de mayo de 2009, en la que concluye afirmando que Los vivos y los muertos es «un libro tan interesante por el 
contenido como por una técnica narrativa que contiene lo mejor de la tradición norteamericana» (2009).

Son muchos los ejemplos que nos muestran que la noción de literatura nacional ya no tiene una real vigencia en América latina, y muchos estudiosos consideran más pertinente y productivo hablar a este respecto de literatura global o transterritorial. Esto llevó a María Ángeles Grande Rosales a definir el nomadismo como un «rasgo característico de la literatura actual, toda vez que lo nacional deja de ser una categoría territorial en un mundo de cultura global» (2017: 53). En este proceso de globalización que estamos viviendo y en el caso que nos interesa, el de Bolivia, es probable que el calificativo que mejor corresponda sea el de literatura escrita por bolivianos y no el de literatura boliviana. Esta última expresión predefine en cierto sentido las obras haciéndolas entrar en un molde cultural, geográfico, social determinado y por ello de cierta manera reductor. Referirse en cambio a una literatura escrita por bolivianos no presupone la adscripción a ninguna norma, a ninguna geografía previas y corresponde incluso a una evolución de la literatura que supera el caso de Bolivia, como lo afirma Jorge Volpi que se refiere ya no a la categoría literatura nacional sino a la categoría de literatura continental:

La especificidad de la literatura hispanoamericana, en nuestros días, es sólo una ilusión: los narradores hispanoamericanos han dejado de escribir sobre los mismos temas, no responden a realidades particularmente cercanas (o, en todo caso, no tienen demasiada posibilidad de comprobarlo), los lectores de cada país no se sienten particularmente identificados con los escritores hispanoamericanos (pueden leer con más facilidad e igual complicidad a un autor europeo o norteamericano) y los escritores de esta parte del mundo se sienten parte de muchas otras tradiciones literarias además de la hispanoamericana (2008: 111-112).

11 La extraterritorialidad, el nomadismo que caracterizan la literatura de las dos últimas décadas no solamente los encontramos en las trayectorias personales de los escritores, sino sobre todo en su escritura, en su manejo del espacio en la ficción, en el sistema de referencias intertextuales, en la definición y caracterización de sus personajes y en la forma misma de sus obras. En el marco limitado de este artículo y al no poder proponer un análisis exhaustivo de la narrativa escrita por bolivianos en lo que va de nuestro siglo, nos detendremos brevemente en dos ejemplos, Tukzon, historias colaterales de Giovanna Rivero, publicada en 2008 y Norte de Edmundo Paz Soldán, publicada en 2011. Estas dos novelas, si bien cuentan historias distintas, tienen más de un punto en común, en particular el hecho de que ambas se ambientan en un espacio de transición -la frontera entre los Estados Unidos y México que constituye un mundo híbrido y angustioso en el que los sueños del desplazado, del inmigrante, se transforman en pesadillas- para relatar trayectorias de personajes desarraigados, alienados, «intensamente extraños» como lo escribe Claudia Bowles en la contratapa de la Tukzon, historiales colaterales de Giovanna Rivero.

12 En Norte, novena novela de Edmundo Paz Soldán que consta de 5 partes y de 33 capítulos, teniendo en cuenta que el último es el epílogo -lo que no deja de ser simbólico si se toma en cuenta el hecho de que uno de los personajes centrales, el asesino serial, se llama Jesús y es ejecutado en el capítulo 33-, cada capítulo lleva así una indicación geográfica, la gran mayoría de las veces el nombre de una ciudad ${ }^{3}$, al que se suma a veces el nombre del estado donde se encuentra, y una indicación temporal. A estas indicaciones se suman por supuesto numerosas referencias espaciales integradas en el relato. Esta multiplicidad de espacios es el reflejo de la movilidad o de la 
inestabilidad de los personajes, en particular de Jesús, el Railroad Killer. El espacio fronterizo es pues el espacio del desarraigo, de los tráficos delictivos de todo tipo y de la violencia más absurda e indefinible, a imagen de los innumerables asesinatos de mujeres que ocurren en Ciudad Juárez, a los que alude el personaje del policía americano de origen mejicano, Rafael Fernández.

Norte se compone por otra parte de tres series de capítulos que alternan sin orden preciso, aunque cada una de ellas se desarrolla en un orden cronológico. La primera serie en un orden cronológico y no en el orden de aparición en la novela, se compone de 5 capítulos (1-3, 2-2, 3-3, 4-1 y 5-1) y se focaliza en el personaje de Martín Ramírez, un inmigrante mejicano que entró ilegalmente en el territorio estadounidense en los años 1930, y que se pasó más de treinta años en los hospitales siquiátricos antes de ser considerado, demasiado tarde, como un gran dibujante. Sus desplazamientos se limitan a los hospitales siquiátricos y a dos ciudades, Stockton y Auburn, situadas ambas en California y distantes la una de la otra de unos $130 \mathrm{~km}$. La segunda serie consta de 18 capítulos (1-1, 1-4, 1-5, 1-6, 2-3, 2-4, 2-5, 2-7, 3-2, 3-5, 3-6, 4-3, 4-4, 4-6, 5-2, 5-4 y 5-7) -a los que se suma en parte el epílogo- y se focaliza en el personaje de Jesús, un adolescente mejicano que se transforma en el primer capítulo en un asesino serial, el famoso Railroad Killer. Sus desplazamientos son muy numerosos como lo podemos observar con las indicaciones espaciales que encabezan los capítulos de esta serie, y se puede decir que excepción hecha de Stockton y Auburn, el personaje de Jesús recorre todos los espacios que se nombran en la novela. La tercera serie en el orden cronológico consta de 9 capítulos (1-2, 2-1, 2-6, 3-1, 3-4, 4-2, 4-5, 5-3 y 5-6) -a los que se suma en parte el epílogo- y se focaliza en Michelle, una joven estudiante de origen boliviano que vive una relación muy complicada con su ex profesor, Fabián.

Es interesante observar aquí que todos los capítulos de la tercera serie transcurren en un mismo espacio, la ciudad de Landslide, espacio al que acude también Jesús en tres oportunidades -1985, 1997 y 1999-, nueve años antes de que Michelle se instale en la ciudad. Michelle intenta abrirse un camino en el sector del cómic y su destino se cruza con el de los personajes de las dos primeras series, sin que sus trayectorias lleguen sin embargo a cruzarse. Esta ciudad de Landslide, además de ser el espacio que más a menudo se nombra en la novela, es también el único en el que coinciden las tres tramas. En Landslide se organiza así en 2009 una retrospectiva de la obra de Martín Ramírez a la que asiste Michelle (cap. 4-5); más de dos décadas antes, en 1985, Jesús había cometido uno de sus primeros asesinatos, una muchacha de 18 años en esa misma ciudad (cap.1-6); allí es también donde, en 1997, el ranger Rafael Fernández identifica a Jesús como el asesino serial (cap. 3-6) y donde el mismo Jesús es arrestado en 1999 (cap. 5-7). Más allá de la importancia cuantitativa dada a este espacio, Landslide tiene la particularidad de que, al igual que la ciudad de Rodeo en esta misma novela, o Madison en Los vivos y los muertos, es una ciudad ficticia. Dicho de otro modo, Landslide se sitúa en Texas en la ficción, es decir en un mapa en el que las distancias entre los lugares mencionados corresponden a las de los mapas, pero que no existe en ningún mapa ${ }^{4}$. De esta manera, Landslide, al igual que Madison en Los vivos y los muertos, o que Río Fugitivo en varias novelas anteriores (La materia del deseo, Sueños digitales, El deliro de Turing) es una suerte de laboratorio de la ficción para Paz Soldán. En esta misma serie, a través de un amigo, Sam, Michelle conoce la historia de Jesús, el Railroad Killer, e imagina un cómic que se inspira de la historia de este asesino serial (2011: 258-260). El lector tiene acceso a la sinopsis de este cómic que se presenta bajo la forma de una 
página cuyos caracteres son diferentes y que está integrada en uno de los últimos capítulos de la novela.

Esta serie de capítulos que se focalizan en Michelle y que se desarrollan en su totalidad en un espacio ficticio, es la única cuyo personaje central es también narrador homodiegético. Se trata de la serie cronológicamente más cercana al presente del lector y es también en esta serie donde se establece el vínculo entre los distintos destinos de los personajes, entre las distintas historias. Este espacio ficticio, Landslide deslizamiento de tierra en español, lo que podría simbolizar la inestabilidad de los personajes- es el que le da unidad a la novela que viene a ser así una especie tríptico del desarraigo, simbolizado aquí por Michelle, una joven boliviana cuyo padre quiere volver a Bolivia, que al principio de la novela quiere dibujar una historia de zombis y ponerle un título poco original, Los muertos vivos - referencia intertextual indirecta a la anterior novela de Paz Soldán-, y que en las últimas páginas parece haber encontrado «la base de [su] relato» (2011: 260), inspirado a la vez de su propia historia, de la del Railroad Killer y de la de Martín Ramírez, un relato que podría ser el de la novela que estamos leyendo. El cómic al que se dedica Michelle establece un vínculo indirecto entre Norte y la novela de Giovanna Rivero, Tukzon. Historias colaterales, publicada en 2008, en la medida en que esta novela fue adaptada al cómic en 2011, año de publicación de Norte.

Tukzon. Historias colaterales se ambienta por su parte en un espacio similar, principalmente los estados del sur de los Estados Unidos, aunque la geografía sea más amplia y abarque hasta Nueva York, en particular en un capítulo titulado «Other voices» que se sitúa en el momento del atentado contra las torres gemelas. Se plantea desde las primeras páginas de Tukzon una interrogación relativa al género narrativo al que pertenece esta obra, ¿novela?, ¿cuento?, ¿otra cosa? En la tapa del libro, la editorial La Hoguera define el libro como «Novela híbrida», calificativo que Luis Horacio Heredia pone en tela de juicio de manera algo discutible en la reseña posteada en 2010 por Marcelo Paz Soldán en Ecdótica, omitiendo al parecer los vínculos intertextuales que se tejen entre los diferentes capítulos de la novela:

Por alguna razón la editorial decidió presentar el libro como «novela híbrida», aunque en mi opinión cada una de las historias, incluso cuando puede existir cierta relación o conexión entre algunas de ellas, son completamente autónomas, lo que les otorga su indudable carácter de cuentos, narraciones cortas muy bien estructuradas (2010).

17 En una reseña anterior publicada asimismo por Ecdótica, Paz Soldán aborda a su vez la cuestión del género al que pertenece este libro de Giovanna Rivero, afirmando por su parte que el libro empieza como un libro de cuentos que se va transformando en novela:

Los cuentos de Tukzon van, de a poco, armando una novela: la historia de una periodista de una revista freak, a la que se le ha pedido escribir un reportaje sobre los «coyotes». Tukzon transcurre en un Estados Unidos en el que el futuro ya es el presente: no es un libro de ciencia ficción, pero sí uno sobre, entre otras cosas, el impacto del imaginario de la ciencia ficción en la vida cotidiana.

Tukzon. Historias colaterales consta pues de 15 capítulos, numerados de 0 a 14, como si ese capítulo 0 fuera el capítulo piloto de una serie televisiva en los cuales se van sucediendo diferentes episodios y diferentes espacios, desde Tucson y el desierto de Arizona hasta Nueva York, pasando por Miami. En dichos espacios van apareciendo numerosos personajes extraños, ambiguos, como Ariadna Nemesis o el hombre desnudo, el 
agente $\mathrm{H}$, que se esconde en el departamento de la narradora llamada $\mathrm{G}$-inicial del nombre de la autora- y que le revela el tráfico (cap. 4 y cap. 9), o el de esa adolescente que muere en el atentado de las torres gemelas en capítulo 6 titulado «Other voices». Esta «novela híbrida», imposible de resumir sin traicionar la complejidad de la trama, como lo escribe Paz Soldán, narra pues la historia de una periodista boliviana enviada a los Estados Unidos por la revista latinoamericana Mother Fucker para realizar un reportaje sobre los «coyotes». Esto genera inevitablemente el malentendido con el cual se inicia la novela, porque la periodista y narradora piensa inicial y equivocadamente que se trata de un reportaje sobre los animales y no sobre aquellos individuos que hacen de intermediarios y de guías para que los emigrantes puedan cruzar clandestinamente la frontera. En realidad se trata incluso de un reportaje sobre un tráfico de órganos organizado en el estado de Arizona, en el cual están implicados los coyotes y cuyas víctimas son los inmigrantes ilegales, los «pachucos»:

Ellas también están involucradas en el horror. Todo el pueblo está de parte del texano. 0 mejor dicho, el texano y todo el pueblo están de parte de alguien más. ¿A dónde estarán exportando los órganos? ¿En qué cuerpos, cuerpos de su cuerpo, estará habitando la mexicana? Claro, desde acá es fácil desaparecer a la gente porque la mayoría son pachucos, de plano nacidos muertos, si me entiende.

El contacto de la periodista es un personaje misterioso, Ariadna Nemesis, quien la tiene que guiar en el desierto de Arizona. Allí se descubre una conspiración espantosa en la cual participan estrellas de Hollywood, en particular Nicole Kidman, que consiste en mantener en vida al presidente de los Estados Unidos, para lo cual se necesitan órganos nuevos.

21 Como lo podemos observar, ambas novelas se ambientan en un espacio que está muy lejos de ser un espacio soñado. La mayoría de los personajes son desarraigados, desplazados, y sus trayectorias desmienten todo tipo de idealización. La extraterritorialidad, vector de apertura hacia el mundo, es reveladora de un mundo que, por ser más abierto, es también mucho más complejo e inasible. El Norte, ese espacio que pudo o puede ser un espacio soñado por aquellos que huyen de la pobreza, como los pachucos, se transforma en cárcel para algunos, como Martín Ramírez, o como un espacio distópico dominado por una violencia que cobra un sinfín de formas que rebasa las fronteras.

\section{BIBLIOGRAFÍA}

CALABUIG Ernesto (2009), «Los vivos y los muertos. Edmundo Paz Soldán», en línea en El Cultural: <https://www.elcultural.com/revista/letras/Los-vivos-y-los-muertos/25349>. FISBACH Erich (2010), Tradition et modernité dans l'œuvre d'Edmundo Paz Soldán, Angers: Presses Universitaires d'Angers.

FISBACH Erich (2011), «Les modalités narratives de l'écriture du mal dans Los vivos y los muertos», E. Ramos Izquierdo (ed.), Autour des écritures du mal, México / Paris: Rilma 2 \& ADEHL, 79-87. 
GRANDE ROSALES María Ángeles (2017), «Géneros móviles y nomadismo literario en la era de la posficción», L. Albuquerque-García, J.-L. García Barrientos y R. Álvarez Escudero (eds), Escritura y teoría en la actualidad, Madrid: Consejo Superior de Investigaciones Científicas.

HEREDIA Luis Horacio (2010), «Acerca de tres libros de Giovanna Rivero», en línea en Ecdótica: <http://www.ecdotica.com/2010/08/24/acerca-de-tres-libros-de-giovanna-rivero/>.

MATTO Javier (2016), «Entrevista a Giovanna Rivero», en línea en La Voz: <https://

www.lavoz.com.ar/ciudad-equis/giovanna-rivero-los-adolescentes-son-mi-maquina-del-tiempo>.

MONTOYA JUÁREZ Jesús (2011), «Arqueologías del presente: cuerpos y escrituras en Los vivos y los muertos», E. Ramos Izquierdo (ed.), Autour des écritures du mal, México / Paris, Rilma 2 \& ADEHL, 61-77.

MONTOYA JUÁREZ Jesús (2017), «De Río Fugitivo a Iris: poshumanismo, forma y discurso en la ficción reciente de Edmundo Paz Soldán», Revista El taco en la brea, revista del Centro de Investigaciones Teórico-literarias -CEDINTEL_ FHUC / UNL, 4(6), <https://bibliotecavirtual.unl.edu.ar/ publicaciones/index.php/ElTacoenlaBrea/article/view/6972/10167>.

NOGUEROL Francisca (2008), «Narrar sin fronteras», J. Montoya Juárez \& Á. Esteban (eds), Entre lo local y lo global. La narrativa latinoamericana en el cambio de siglo (1900-2006), Iberoamericana Editorial Vervuert.

PALMA Ricardo (1964), «El Manchay Puito», Tradiciones peruanas completas [1872], Madrid: Aguilar. PAZ SOLDÁN Edmundo (2009), Los vivos y los muertos, Madrid: Alfaguara.

PAZ SOLDÁN Edmundo (2011), Norte, Barcelona: Mondadori.

PAZ SOLDÁN Edmundo (2012), «Tres postales», Bolivia a toda costa. Crónicas de un país de ficción (Selección prólogo de Fernando Barrientos), La Paz: Editorial El Cuervo.

RIVERO Giovanna (2008), Tukzon, historias colaterales, Santa Cruz, Editorial La Hoguera.

RIVERo Giovanna (2012), «Berlín Santa Cruz», F. Barrientos (selección y prólogo), Bolivia a toda costa. Crónicas de un país de ficción, La Paz: Editorial El Cuervo.

SÁNCHEZ Pablo (2016), «Después de cruzar la frontera se llega al límite: Norte, de Edmundo Paz Soldán», Anuario de Estudios Americanos, 73(2), <http://estudiosamericanos.revistas.csic.es/ index.php/estudiosamericanos/article/view/686>.

STEINER George (2002), Extraterritorial. Ensayos sobre literatura y la renovación lingüística (trad. Edgardo Russo), Madrid: Ediciones Siruela.

TABOADA TERÁN Néstor (2001), Manchay Puytu, el amor que quiso ocultar Dios (6ta edición) [1ra edición, Buenos Aires, Sudamericana, 1977], Cochabamba: Ed. El Pájaro de fuego.

VARGAS LLOSA Mario (2000), «Las culturas y la globalización», en línea en El País: <https:// elpais.com/diario/2000/04/16/opinion/955836005_850215.html>.

VOLPI Jorge (2008), «Narrativa hispanoamericana, INC.», J. Montoya Juárez \& Á. Esteban (eds), Entre lo local y lo global. La narrativa latinoamericana en el cambio de siglo (1900-2006), Iberoamericana Editorial Vervuert.

\section{NOTAS}

1. Felipe Delgado de Jaime Sáenz se publicó en 1979. 
2. La niña de sus ojos de Antonio Díaz Villamil se publicó en 1948.

3. Un solo capítulo, el capítulo 4 de la quinta parte, no lleva el nombre de ninguna ciudad sino el de dos estados estadounidenses.

4. En un intercambio de mails, Edmundo Paz Soldán me confirmó que Landslide es una ciudad ficticia inspirada por la ciudad de Austin en la que vivió.

\section{RESÚMENES}

Desde la recuperación de la democracia en Bolivia en 1982 tras décadas de gobiernos autoritarios, la imagen de una literatura encerrada en sus fronteras se diluye. Los novelistas bolivianos participan plenamente de la globalización; varios de ellos, a imagen de Edmundo Paz Soldán, residen en el extranjero, y abordan y desarrollan desde allí temáticas muy alejadas del localismo o del regionalismo tradicionales. Este estudio se concentra en dos ejemplos, Norte de Paz Soldán y Tukzon, historias colaterales de Giovanna Rivero, dos novelas que se ambientan en un espacio de transición - la frontera entre los Estados Unidos y México- para relatar historias de personajes desarraigados, alienados, «intensamente extraños» como lo escribe Claudia Bowles a propósito de la novela de Giovanna Rivero. El estudio en particular en los recursos narrativos empleados para construir este universo híbrido y de pesadilla.

Depuis la récupération de la démocratie en Bolivie en 1982 après plusieurs décennies de gouvernements autoritaires, l'image d'une littérature enfermée dans ses frontières se dilue. Les romanciers boliviens participent pleinement de la globalisation; plusieurs d'entre eux, à l'instar d'Edmundo Paz Soldán, résident à l'étranger, et abordent et développent depuis cet espace autre des thématiques très éloignées du localisme ou du régionalisme traditionnels. Ce travail se concentre sur deux exemples, Norte d'Edmundo Paz Soldán et Tukzon, historias colaterales de Giovanna Rivero, deux romans qui prennent pour cadre un espace de transition - la frontière entre les États-Unis et le Mexique - pour raconter des histoires de personnages déracinés, aliénés, « intensément étranges » comme l'écrit Claudia Bowles à propos du roman de Giovanna Rivero. Cet article se focalise en particulier sur les procédés narratifs mis en place afin de construire cet univers hybride et cauchemardesque.

Since the recovery of democracy in Bolivia in 1982 after several decades of authoritarian governments, the image of a literature enclosed within its boundaries has been diluting. Bolivian novelists are fully participating in globalisation. Many of them, like Edmundo Paz Soldán, reside abroad and are approaching and developing other themes which are very far from traditional localism or regionalism. This work focuses on two examples, Edmundo Paz Soldán's Norte and Giovanna Rivero's Tukzon, historias colaterales, two novels which are set in a transition space-the border between the United States and Mexico-to tell stories of uprooted, alienated, "intensely strange" characters as Claudia Bowles writes about Giovanna Rivero's novel. This article focuses in particular on the narrative techniques used to create this hybrid and nightmarish universe. 
ÍNDICE

Mots-clés: Edmundo Paz Soldán, Giovanna Rivero, globalisation, roman latino-américain, Bolivie Palabras claves: Edmundo Paz Soldán, Giovanna Rivero, globalización, novela latinoamericana, Bolivia

Keywords: Edmundo Paz Soldán, Giovanna Rivero, globalization, latin american novel, Bolivia

\section{AUTOR}

ERICH FISBACH

3L.AM - UPRES EA 4335, Univ. Angers, SFR Confluences 4201, France 